\title{
Zeroes of Slice Monogenic Functions
}

\author{
Yan Yang and Tao Qian \\ School of Mathematics and Computational Science, Sun Yat-Sen University, and \\ Department of Mathematics, Faculty of Science and Technology, University of Macau \\ E-mail: mathyy@sina.com, fsttq@umac.mo
}

\begin{abstract}
In this article, we study structure of zeroes of power series with Clifford algebravalued coefficients. Especially, if it has paravector-valued coefficients, we obtain some sufficient and necessary conditions of power series that have zeroes, as well as a method to compute the zeroes if exist.
\end{abstract}

keywords: Clifford algebra, Zero sets

AMS subject classification: 30G35, 32A05

\section{Introduction}

There has been a continuing study on zeroes of polynomials in quaternions and octonions. Niven in $[3,4]$ first studied zeroes of quaternionic polynomials. The fundamental theorem of algebra for quaternionic polynomials was established by Eilenberg and Niven in [5]. Ever since then the theorem has been re-proved by using a number of different methods. In [6], the authors use a constructive method to prove it. In [7], the authors introduce a regular multiplication in order to prove the theorem and also give factorization of regular functions. In [8], structure of zeroes of polynomials is studied and a topological proof of the fundamental theorem for both quaternionic and octonionic variables is given. Besides those, multiplicity of zeroes of quaternionic polynomials is studied in [9]. In [10] the authors study zeroes of quaternionic and octonionic Laurent series with real coefficients using a geometrical method. To the authors knowledge, in the Clifford algebra setting for higher dimensions, there have been no so similar results. In [13] the authors independently study zeros of Clifford polynomials. Using a technical method, [13] introduces a one-to-one correspondence between Clifford polynomials of real-coefficients with complex polynomials of real-coefficients. They further extend the results to Laurent series with real coefficients in [14]. Algorithms of zeros are actually given in [13] and [14].

In $[11,12]$, the authors introduce slice monogenic functions that include polynomials and power series in the Clifford algebra setting as particular cases. Fundamental properties of zeroes of Clifford power series on a special class of domains are studied.

\footnotetext{
${ }^{1}$ This project sponsored by the National Natural Science Funds for Young Scholars (No. 10901166) and by Research Grant of University of Macau on Applications of Hyper-Complex Analysis (cativo: 7560), the FDCT project on Clifford and Harmonic Analysis (014/2008/A1)
} 
By adopting the setting of slice monogenic functions, in the present work, structure of zeroes of slice monogenic functions is studied. Some of the results are overlap with the above mentioned literature but with different methodology for the proofs. As main contributions of this work we obtain sufficient and necessary conditions for slice monogenic functions with paravector-valued coefficients to have zeros, and we have practical methods to find the zeroes if they exist.

We first give some basic knowledge in relation to Clifford algebra ([1,2]). Let $\mathbf{e}_{1}, \ldots, \mathbf{e}_{m}$ be basic elements satisfying $\mathbf{e}_{i} \mathbf{e}_{j}+\mathbf{e}_{j} \mathbf{e}_{i}=-2 \delta_{i j}$, where $\delta_{i j}=1$ if $i=j$; and $\delta_{i j}=0$ otherwise, $i, j=1,2, \cdots, m$. Let

$$
\mathbf{R}^{m}=\left\{\underline{x}=x_{1} \mathbf{e}_{1}+\cdots+x_{m} \mathbf{e}_{m}: x_{j} \in \mathbf{R}, j=1,2, \cdots, m\right\}
$$

be identical with the usual Euclidean space $\mathbf{R}^{m}$, and

$$
\mathbf{R}_{1}^{m}=\left\{x=x_{0} \mathbf{e}_{0}+\underline{x}: x_{0} \in \mathbf{R}, \underline{x} \in \mathbf{R}^{m}\right\} \text {, where } \mathbf{e}_{0}=1 \text {. }
$$

An element in $\mathbf{R}^{\mathbf{m}}$ is called a vector, and an element in $\mathbf{R}_{1}^{m}$ is called a paravector. A paravector $x \in \mathbf{R}_{1}^{m}$ consists of a scalar part and a vector part. We use the denotions

$$
x_{0}=\operatorname{Sc}(x), \underline{x}=\operatorname{Vec}(x) .
$$

The real (or complex) Clifford algebra generated by $\mathbf{e}_{1}, \mathbf{e}_{2}, \cdots, \mathbf{e}_{m}$, denoted by $\mathbf{R}^{(m)}$ (or $\mathbf{C}^{(m)}$ ), is the associative algebra generated by $\mathbf{e}_{1}, \mathbf{e}_{2}, \cdots, \mathbf{e}_{m}$ over the real (or complex) field $\mathbf{R}$ (or $\mathbf{C}$ ). A general element in $\mathbf{R}^{(m)}$ (or $\mathbf{C}^{(m)}$ ), therefore, is of the form $x=$ $\sum_{S} x_{S} \mathbf{e}_{S}$, where $\mathbf{e}_{S}=\mathbf{e}_{i_{1}} \mathbf{e}_{i_{2}} \cdots \mathbf{e}_{i_{l}}, x_{S} \in \mathbf{R}$ (or $\mathbf{C}$ ), and $S$ runs over all the ordered subsets of $\{1,2, \cdots, m\}$, namely

$$
S=\left\{1 \leq i_{1}<i_{2}<\cdots<i_{l} \leq m\right\}, \quad 1 \leq l \leq m
$$

We use $[x]_{0}$ to denote the scalar part of a Clifford number $x \in \mathbf{R}^{(m)}$.

We define the conjugation of $\mathbf{e}_{S}$ to be $\overline{\mathbf{e}}_{S}=\overline{\mathbf{e}}_{i_{l}} \cdots \overline{\mathbf{e}}_{i_{1}}, \overline{\mathbf{e}}_{j}=-\mathbf{e}_{j}$. This induces the Clifford conjugate $\bar{x}=x_{0}-\underline{x}$ of a paravector $x=x_{0}+\underline{x}$.

The product between $x$ and $y$ in $\mathbf{R}_{1}^{m}$, denoted by $x y$, is split into three parts: a scalar part, a vector part and a bivector part, that is

$$
x y=\left(x_{0} y_{0}+\underline{x} \cdot \underline{y}\right)+\left(x_{0} \underline{y}+y_{o} \underline{x}\right)+\underline{x} \wedge \underline{y},
$$

where

$$
\begin{gathered}
\underline{x} \cdot \underline{y}=-\sum_{i=1}^{m} x_{i} y_{i}, \\
\underline{x} \wedge \underline{y}=\sum_{i=1}^{m} \sum_{j=i+1}^{m}\left(x_{i} y_{j}-x_{j} y_{i}\right) \mathbf{e}_{i} \mathbf{e}_{j} .
\end{gathered}
$$

In particular,

$$
x x=x_{0}^{2}-\sum_{i=1}^{m} x_{i}^{2}+2 x_{0} \underline{x}=2 x_{0} x-|x|^{2},
$$


where

$$
|x|^{2}=x \bar{x}=\sum_{i=0}^{m} x_{i}^{2} .
$$

It is easy to see that $\left|x^{n}\right|=|x|^{n}$.

In Clifford analysis, the differential operator

$$
\frac{\partial}{\partial x}=\sum_{n=0}^{m} \frac{\partial}{\partial x_{n}} e_{n}
$$

called the Cauchy-Riemman operator, is used to define monogenic functions (regular functions) in higher dimensions. In the Clifford algebra setting, the fundamental tools such as Cauchy theorem, Cauchy integral formula, Taylor and Laurent series all exist. However, some basic functions such as polynomials are not monogenic. In order to modify this, H. Leutwiler studied the modified quaternionic analysis and its higher dimensional extensions in [17]. Another way is inspired by C. G. Cullen in [18]. G. Gentili and D. C Struppa offered an alternative definition for regular functions and then studied the theory in $[19,20]$. It is now further extended to higher dimensions by F. Colombo, I. Sabadini and D. C Struppa. Under this new setting, a similar fundamental theory also exists. For details, please see $[11,12]$.

In order to give the definition of slice monogenic function, we use $S^{m-1}$ to denote the $(m-1)$-dimensional unit sphere in $\mathbf{R}^{m}$. That is, $S^{m-1}=\left\{\underline{I} \in \mathbf{R}^{m}, \underline{I}^{2}=-1\right\}$. Denote

$$
L_{\underline{I}}=\left\{\alpha+\beta \underline{I} \in \mathbf{R}_{1}^{m}, \alpha, \beta \in R, \underline{I} \in S^{m-1}\right\} .
$$

and

$$
\operatorname{Re}[\alpha+\beta \underline{I}]=\alpha, \operatorname{Im}[\alpha+\beta \underline{I}]=\beta \text {. }
$$

Definition 1.1 ${ }^{[12]}$ Let $U \subseteq \mathbf{R}_{1}^{m}$ be an open set and $f: U \rightarrow \mathbf{R}^{(m)}$ be a real differentiable function. Let $\underline{I} \in S^{m-1}$ and $f_{\underline{I}}$ be the restriction of $f$ to the complex plane $L_{\underline{I}}=\mathbf{R}+\underline{I} \mathbf{R}$. We say that $f$ is a left slice monogenic function (in short slice monogenic function) if for every $\underline{I} \in S^{m-1}$,

$$
\frac{1}{2}\left(\frac{\partial}{\partial u}+\underline{I} \frac{\partial}{\partial v}\right) f_{\underline{I}}(u+\underline{I} v)=0
$$

Note 1.1 Similarly, we can define right slice monogenic functions if $f_{\underline{I}}(u+\underline{I} v) \frac{1}{2}\left(\frac{\partial}{\partial u}+\right.$ $\left.\underline{I} \frac{\partial}{\partial v}\right)=0$.

Since for all $\underline{I} \in S^{m-1}$, we have

$$
\frac{1}{2}\left(\frac{\partial}{\partial u}+\underline{I} \frac{\partial}{\partial v}\right)(u+v \underline{I})^{n}=0 .
$$

Hence $x^{n}$ is slice monogenic. Similarly, polynomials and power series in its convergent set are also slice monogenic. 
For slice monogenic functions, a notion of derivative of $f$ has been introduced.

Definition 1.2 ${ }^{[12]}$ Let $U \subseteq \mathbf{R}_{1}^{m}$ be an open set in $\mathbf{R}_{1}^{m}$ and $f$ be a left slice monogenic function in $U$. It slice monogenic derivative is defined by

$$
\frac{\partial f}{\partial x}(x)=\partial_{\underline{I}} f(x), x=u+\underline{I} v, v \neq 0 ; \text { or } \partial_{u} f(u), u \in \mathbf{R},
$$

where $\partial_{\underline{I}}=\frac{1}{2}\left(\frac{\partial}{\partial u}-\underline{I} \frac{\partial}{\partial v}\right)$.

Theorem 1.1 ${ }^{[12]}$ Let $f: B(0, R) \rightarrow \mathbf{R}^{(m)}$ be a slice monogenic function. It can be represented in power series

$$
f(x)=\sum_{n=0}^{\infty} x^{n} \frac{1}{n !} \frac{\partial^{n} f}{\partial u^{n}}(0)
$$

converging on $B(0, R)$.

In the following the so-called Clifford-Heaviside functions

$$
P^{ \pm}(\underline{x})=\frac{1}{2}\left(1 \pm \mathbf{i} \frac{\underline{x}}{|\underline{x}|}\right)
$$

will play an important role (see [15] and [16]). Introducing spherical coordinates in $\mathbf{R}^{m}$, we have $\underline{x}=r \underline{I}, r=|\underline{x}| \in[0, \infty), \underline{I} \in S^{m-1}$. Thus,

$$
P^{ \pm}(\underline{I})=\frac{1}{2}(1 \pm \mathbf{i} \underline{I}) .
$$

They are self adjoint mutually orthogonal primitive idempotents:

$$
P^{+}(\underline{I})+P^{-}(\underline{I})=1, P^{+}(\underline{I}) P^{-}(\underline{I})=P^{-}(\underline{I}) P^{+}(\underline{I})=0,\left(P^{ \pm}(\underline{I})\right)^{2}=P^{ \pm}(\underline{I}) .
$$

Furthermore, we have $P^{ \pm}(\underline{I})\left(x_{0}+r \underline{I}\right)=P^{ \pm}(\underline{I})\left(x_{0} \mp \mathbf{i} r\right)$.

Acknowledgement: The authors would like to express their sincere thanks to Prof I. Sabadini and Prof F. Vlacci for providing us their recent papers on the related studies.

\section{Structure of the Zeroes of slice monogenic functions}

In this section, we will consider the zeroes of slice monogenic function

$$
f(x)=\sum_{n=0}^{\infty} x^{n} a_{n}, a_{n} \in \mathbf{R}^{(m)} .
$$

In [13], we computed that if $x=x_{0}+\underline{x} \in \mathbf{R}_{1}^{m}$, then

$$
x^{n}=A_{n}(x) x+B_{n}(x), n=1,2, \cdots
$$

where $A_{n}$ and $B_{n}$ are real-valued functions of $x$ defined by the recusive formulas:

$$
\begin{aligned}
& A_{n+1}(x)=2 x_{0} A_{n}(x)-|x|^{2} A_{n-1}(x) \\
& B_{n+1}(x)=-|x|^{2} A_{n}(x),
\end{aligned}
$$


where

$$
\begin{aligned}
& A_{1}(x)=1 \\
& A_{2}(x)=2 x_{0} \\
& B_{1}(x)=0 \\
& B_{2}(x)=-|x|^{2} .
\end{aligned}
$$

Denoting $A_{0}(x)=0, B_{0}(x)=1$, therefore,

$$
\begin{aligned}
f(x) & =\sum_{n=0}^{\infty}\left[A_{n}(x) x+B_{n}(x)\right] a_{n} \\
& =x\left[\sum_{n=0}^{\infty} A_{n}(x) a_{n}\right]+\left[\sum_{n=0}^{\infty} B_{n}(x) a_{n}\right] \\
& =x A(x)+B(x) .
\end{aligned}
$$

Note 2.1 As we have known in [13], given any $x \in \mathbf{R}_{1}^{m}, A_{i}(x)$ and $B_{i}(x)$ can be expressed as functions of its scalar part $x_{0}$ and the modulus of its vector part $|\underline{x}|$. Thus, we have

Proposition 2.1 Let $w_{1}=\alpha+\beta \underline{I_{1}}$ and $w_{2}=\alpha+\beta \underline{I_{2}}$ be two different paravectors, then $A_{i}\left(w_{1}\right)=A_{i}\left(w_{2}\right), B_{i}\left(w_{1}\right)=B_{i}\left(w_{2}\right)$ and hence $A\left(w_{1}\right)=A\left(w_{2}\right), B\left(w_{1}\right)=B\left(w_{2}\right)$. Particularly, we have $A(w)=A(\bar{w}), B(w)=B(\bar{w})$.

Definition 2.1 ${ }^{[13]}$ Let $w_{1}=\alpha+\beta \underline{I_{1}}$ and $w_{2}=\alpha+\beta \underline{I_{2}}$ be two different paravectors, then they are said to be spherical conjugate to each other.

Let

$$
f(x)=\sum_{n=0}^{\infty} x^{n} a_{n}\left(a_{n} \in \mathbf{R}^{(m)}\right)
$$

be a slice monogenic function converging in $B(0, R)$. Using the Proposition 2.1, we can obtain the following result that has been proved in [11] by a different way.

Theorem 2.1 ${ }^{[11]}$ Let $f(x)$ be slice monogenic as given in (1). Assume that $w_{1}=\alpha+\beta \underline{I_{1}}$ and $w_{2}=\alpha+\beta \underline{I_{2}}$ are two different zeroes of $f(x)$, where $\underline{I_{1}}, \underline{I_{2}} \in S^{m-1}$ and $\alpha^{2}+\beta^{2}<\overline{R^{2}}$. Then any paravector $w=\alpha+\beta \underline{I}$ for all $\underline{I} \in S^{m-1}$ being spherical conjugate to $w_{1}$ is also a zero of it.

Proof If $f\left(w_{1}\right)=0$ and $f\left(w_{2}\right)=0$, then we have

$$
f\left(w_{1}\right)=w_{1} A\left(w_{1}\right)+B\left(w_{1}\right)=0,
$$

and

$$
f\left(w_{2}\right)=w_{2} A\left(w_{2}\right)+B\left(w_{2}\right)=0,
$$

using Proposition 2,1, we have $A\left(w_{1}\right)=A\left(w_{2}\right), B\left(w_{1}\right)=B\left(w_{2}\right)$. Using $(2)-(3)$, we have

$$
\left(w_{1}-w_{2}\right) A\left(w_{1}\right)=\beta\left(\underline{I_{1}}-\underline{I_{2}}\right) A\left(w_{1}\right)=0 .
$$


thus $A\left(w_{1}\right)=0$ and then $B\left(w_{1}\right)=0$.

For any $w=\alpha+\beta \underline{I}$, using Proposition 2,1, we have $A(w)=A\left(w_{1}\right), B(w)=B\left(w_{1}\right)$.

Therefore, $f(w)=w A(w)+B(w)=w A\left(w_{1}\right)+B\left(w_{1}\right)=0$. This completes the proof.

Definition 2.2 Let $f(x)$ be slice monogenic as given in (1), then any of its zeroes generating a family of zeroes that are spherical conjugate to each other is called a spherical zero.

From Theorem 2,1, we know that

Corollary 2.1 Let $f(x)$ be slice monogenic as given in (1). Then $\alpha+\beta \underline{I}$ is a spherical zero of $f(x)$ if and only if $\alpha \pm \beta \underline{I}, \beta \neq 0$ are zeroes of it.

Theorem 2.2 Let $f(x)$ be slice monogenic as given in (1) and $I_{1}, \underline{I_{2}}$ be two different units. If $a_{n} \in L_{\underline{I_{1}}}, n=0,1, \cdots$, and $\alpha+\beta \underline{I_{2}}(\beta \neq 0)$ is a zero of $f(x)$. Then $\alpha+\beta \underline{I}$ is a spherical zero of it.

Proof For $f(x)=x A(x)+B(x)$, we have

$$
\begin{aligned}
f\left(\alpha+\beta \underline{I_{2}}\right) & =\left(\alpha+\beta \underline{I_{2}}\right) A\left(\alpha+\beta \underline{I_{2}}\right)+B\left(\alpha+\beta \underline{I_{2}}\right) \\
& =\left[\alpha A\left(\alpha+\beta \underline{I_{2}}\right)+B\left(\alpha+\beta \underline{I_{2}}\right)\right]+\left[\beta \underline{I_{2}} A\left(\alpha+\beta \underline{I_{2}}\right)\right] .
\end{aligned}
$$

Noting that $a_{n} \in L_{\underline{I_{1}}}, n=0,1, \cdots$, we have

$$
\begin{aligned}
& A(x)=\sum_{n=0}^{\infty} A_{n}(x) a_{n} \in L_{\underline{I_{1}}} \\
& B(x)=\sum_{n=0}^{\infty} B_{n}(x) a_{n} \in L_{\underline{I_{1}}} .
\end{aligned}
$$

Letting

$$
\begin{aligned}
& A\left(\alpha+\beta \underline{I_{2}}\right)=x_{1}+y_{1} \underline{I_{1}} \\
& B\left(\alpha+\beta \underline{I_{2}}\right)=x_{2}+y_{2} \underline{I_{1}},
\end{aligned}
$$

we have

$$
\begin{aligned}
f\left(\alpha+\beta \underline{I_{2}}\right) & =\left[\alpha A\left(\alpha+\beta \underline{I_{2}}\right)+B\left(\alpha+\beta \underline{I_{2}}\right)\right]+\left[\beta \underline{I_{2}} A\left(\alpha+\beta \underline{I_{2}}\right)\right] \\
& =\left[\alpha x_{1}+x_{2}+\beta y_{1} \underline{I_{2}} \cdot \underline{I_{1}}\right]+\left[\alpha y_{1} \underline{I_{1}}+\beta x_{1} \underline{I_{2}}+y_{2} \underline{I_{1}}\right]+\beta y_{1} \underline{I_{2}} \wedge \underline{I_{1}} \\
& =0,
\end{aligned}
$$

implying

$$
\begin{aligned}
& \alpha x_{1}+x_{2}+\beta y_{1} \underline{I_{2}} \cdot \underline{I_{1}}=0 \\
& \alpha y_{1} \underline{I_{1}}+\beta x_{1} \underline{I_{2}}+y_{2} \underline{I_{1}}=0 \\
& \beta y_{1} \underline{I_{2}} \wedge \underline{I_{1}}=0 .
\end{aligned}
$$


Since $\beta \neq 0$, it is easily to obtain $y_{1}=0$. Then $y_{2}=0, x_{1}=0$ and $x_{2}=0$. Hence $A\left(\alpha+\beta \underline{I_{2}}\right)=B\left(\alpha+\beta \underline{I_{2}}\right)=0$.

For all $\underline{I} \in S^{m-1}$, we have

$$
\begin{aligned}
f(\alpha+\beta \underline{I}) & =(\alpha+\beta \underline{I}) A(\alpha+\beta \underline{I})+B(\alpha+\beta \underline{I}) \\
& =(\alpha+\beta \underline{I}) A\left(\alpha+\beta \underline{I_{2}}\right)+B\left(\alpha+\beta \underline{I_{2}}\right) \\
& =0 .
\end{aligned}
$$

This completes the proof.

Corollary 2.2 Let $f(x)$ be slice monogenic as given in (1). If $a_{n}=u_{n}+v_{n} \underline{I_{1}} \in L_{\underline{I_{1}}}, n=$ $0,1, \cdots$, and $f$ has non-spherical and non-real zeroes, then the zeroes must belong to $L_{I_{1}}$. Furthermore, there exists a one-to-one correspondence between the zero $\alpha+\beta \underline{I_{1}}$ of $f(\bar{x})$ and the zero $\alpha+\mathbf{i} \beta$ of $f_{1}(z)$, where

$$
f_{1}(z)=\sum_{n=0}^{\infty}\left(u_{n}+\mathbf{i} v_{n}\right) z^{n}
$$

Proof: The first assertion is concluded from Theorem 2.2. To prove the second part, for $x=\alpha+\beta \underline{I_{1}}$, using the properties of $P^{ \pm}$, we have

$$
\begin{aligned}
f\left(\alpha+\beta \underline{I_{1}}\right)=0 & \Longleftrightarrow\left[P^{+}\left(\underline{I_{1}}\right)+P^{-}\left(\underline{I_{1}}\right)\right] f\left(\alpha+\beta \underline{I_{1}}\right)=0 \\
& \Longleftrightarrow P^{+}\left(\underline{I_{1}}\right) \overline{f_{1}(\alpha+\mathbf{i} \beta)}+P^{-}\left(\underline{I_{1}}\right) f_{1}(\alpha+\mathbf{i} \beta)=0 \\
& \Longleftrightarrow P^{+}\left(\underline{I_{1}}\right) \overline{f_{1}(\alpha+\mathbf{i} \beta)}=0 \text { and } P^{-}\left(\underline{I_{1}}\right) f_{1}(\alpha+\mathbf{i} \beta)=0 . \\
& \Longleftrightarrow f_{1}(\alpha+\mathbf{i} \beta)=0 .
\end{aligned}
$$

Theorem 2.3 Let $f(x)$ be a slice monogenic function as given in (1). If $f\left(L_{I_{1}}\right) \subseteq L_{I_{1}}$ and $f\left(L_{I_{2}}\right) \subseteq L_{I_{2}}$, where $\underline{I_{1}}, \underline{I_{2}} \in S^{m-1}$ are two different units, then the coefficients of $f(x)$ are real-valued.

Proof According to Theorem 1.1, for $x=u+v \underline{I}$, we have

$$
f(x)=\sum_{n=0}^{\infty} x^{n} a_{n}
$$

where $a_{n}=\frac{1}{n !} \frac{\partial^{n} f}{\partial u^{n}}(0)$. If $f\left(L_{I_{1}}\right) \subseteq L_{\underline{I_{1}}}$, then $\frac{\partial^{n} f}{\partial u^{n}}(0) \in \underline{I_{1}}$. Hence $a_{n} \in \underline{I_{1}}$. Similarly, if $f\left(L_{I_{2}}\right) \subseteq L_{I_{2}}$, then $a_{n} \in \underline{I_{2}}$. While $\underline{I_{1}} \neq \underline{I_{2}}$, we have $a_{n} \in \mathbf{R}$. This completes the proof.

In [14], we discussed the zeroes of Laurent series with real coefficients and we obtained a one-to-one correspondence relationship between the zeroes of $f(x)$ and those of $f(z)$. That is:

Theorem 2.4 ${ }^{[14]}$ Let $f(x)=\sum_{n=-\infty}^{\infty} a_{n} x^{n},(r<|x|<R)$, be a Laurent series with real coefficients. Then there is a one-to-one correspondence between the zeroes of $\alpha \pm \mathbf{i} \beta$ of $f(z)$ and the spherical zero $\alpha+\beta \underline{I}$ of $f(x)$. 
Particularly, if $f$ has paravector valued coefficients, we have the isolated zero principle: Theorem 2.5 Let $f(x): B(0, R) \rightarrow \mathbf{R}^{(m)}$ be a slice monogenic function with paravector valued coefficients. If $f$ has a accumulation zero point in $B(0, R)$, then $f \equiv 0$ in $B(0, R)$.

We will prove it in next section.

Corollary 2.5 Let $f(x), g(x): B(0, R) \rightarrow \mathbf{R}^{(m)}$ be slice monogenic functions with paravector valued coefficients. If there exists a subset $T \in B(0, R)$ having an accumulation point such that $f=g$ on $T$, then $f \equiv g$ in $B(0, R)$.

Remark: In [12], the identity principle of slice monogenic functions with Clifford algebravalued coefficients has been given. But it needs extra conditions.

\section{Computation of the zeroes of $f(x)$}

In this section, we mainly discuss how to solve the zeroes of slice monogenic function $f(x)=\sum_{n=0}^{\infty} x^{n} a_{n}$ with paravector-valued coefficients.

Firstly, we introduce the function:

$$
f^{c}(x)=\overline{f(\bar{x})}=\sum_{n=0}^{\infty} \overline{a_{n}} x^{n}
$$

Definition 3.1 Define

$$
F(x)=f(x) * f^{c}(x)=\sum_{n=0}^{\infty} x^{n} c_{n}
$$

where $c_{n}=\sum_{k=1}^{n} a_{k} \overline{a_{n-k}}$ for all $n$.

\section{Lemma 3.1}

(I) Let $f(x)=x A(x)+B(x)$, then $f^{c}(x)=\overline{A(\bar{x})} x+\overline{B(\bar{x})}=\overline{A(x)} x+\overline{B(x)}$.

(II) $F(x)$ is a slice monogenic function with real coefficients.

(III) $f(x) * f^{c}(x)=f^{c}(x) * f(x)$.

(IV) $F(z)=f(z) f^{c}(z)$, while $F(x) \neq f(x) f^{c}(x)$.

We have $F(z)=f(z) f^{c}(z)$ by a direct calculation. On the other hand,

$$
\begin{aligned}
F(z) & =f(z) f^{c}(z) \\
& =[z A(z)+B(z)][\overline{A(z)} z+\overline{B(z)}] \\
& =|A(z)|^{2} z^{2}+[A(z) \overline{B(z)}+B(z) \overline{A(z)}] z+|B(z)|^{2} .
\end{aligned}
$$

So another form of $F(x)$ is:

$$
F(x)=|A(x)|^{2} x^{2}+[A(x) \overline{B(x)}+B(x) \overline{A(x)}] x+|B(x)|^{2} .
$$


While

$$
\begin{aligned}
f(x) f^{c}(x) & =[x A(x)+B(x)][\overline{A(x)} x+\overline{B(x)}] \\
& =|A(x)|^{2} x^{2}+[x A(x) \overline{B(x)}+B(x) \overline{A(x)} x]+|B(x)|^{2} .
\end{aligned}
$$

Therefore,

$$
F(x)=f(x) f^{c}(x) \Longleftrightarrow x A(x) \overline{B(x)}=A(x) \overline{B(x)} x .
$$

For $f(x)=\sum_{n=0}^{\infty} x^{n} a_{n}, a_{n} \in \mathbf{R}_{1}^{m}$ and $x=x_{0}+|\underline{x}| \underline{I}$, we have

$$
\begin{aligned}
P^{ \pm}(\underline{I}) f(x) f^{c}(x) P^{ \pm}(\underline{I}) & =P^{ \pm}(\underline{I}) f\left(x_{0} \mp \mathbf{i}|\underline{x}|\right) f^{c}\left(x_{0} \mp \mathbf{i}|\underline{x}|\right) P^{ \pm}(\underline{I}) \\
& =P^{ \pm}(\underline{I}) F\left(x_{0} \mp \mathbf{i}|\underline{x}|\right) P^{ \pm}(\underline{I}) .
\end{aligned}
$$

Therefore,

$$
\begin{aligned}
f(x)=0 & \Longrightarrow P^{ \pm}(\underline{I}) f(x) f^{c}(x) P^{ \pm}(\underline{I})=0 \\
& \Longrightarrow P^{ \pm}(\underline{I}) F\left(x_{0} \mp \mathbf{i}|\underline{x}|\right) P^{ \pm}(\underline{I})=0 \\
& \Longrightarrow F(z)=0 .
\end{aligned}
$$

Note 3.1 Note that $F(z)=0$ is an equation of real coefficients. It, therefore, has complex conjugate roots.

From the above discussion, we can obtain the conclusion as follows:

Proposition 3.1 If $\alpha+\beta \underline{I}, \beta>0$, is a zero (spherical zero or non-real zero) of $f(x)$, then $\alpha \pm \mathbf{i} \beta$ are solutions of $F(z)=0$.

Proof of Theorem 2.5 Suppose $x_{n}+y_{n} \underline{I_{n}},(n=1,2, \cdots)$ are a sequence of non-spherical conjugate zeroes of $f(x)$ and they have an accumulation point $x+y \underline{I}$. Then $x_{n} \pm \mathbf{i} y_{n}$ are a sequence of conjugate zeroes of $F(z)$ and which have an accumulation point $x \pm \mathbf{i} y$. By the isolate zero principal, we know that $F(z) \equiv 0$. Then the coefficients $c_{n}=\sum_{k=1}^{n} a_{n} \overline{a_{n-k}}$ of $F$ vanish. This induces the coefficients $a_{n}$ of $f$ vanish. Hence $f \equiv 0$. These completes the proof.

Remark: In above discussion, if $f(x)=\sum_{n=0}^{\infty} x^{n} a_{n}, a_{n} \in \mathbf{R}^{(m)}$, then from $f\left(x_{0}+|\underline{x}| \underline{I}\right)=$ 0 we cannot get $F\left(x_{0} \mp \mathbf{i}|\underline{x}|\right)=0$. We only obtain $\left[F\left(x_{0} \mp \mathbf{i}|\underline{x}|\right)\right]_{0}=0$ which is an equation of real coefficients. So if $f(x)$ has non-spherical conjugate zeroes which have an accumulation zero, then $[F(z)]_{0}$ has an accumulation point. Thus $[F(z)]_{0} \equiv 0$. But from $[F(z)]_{0} \equiv 0$, we cannot derive $f(x) \equiv 0$.

Next, we will discuss the relationship between the zeroes of $F(z)$ and those of $f(x)$. First we need a Lemma.

Lemma 3.2 Let $f(x)$ be slice monogenic as given in (1). Then the zeroes of $f(x)$ is in one-to-one correspondence with those of $f^{c}(x)$. 
Proof a) If $w=\alpha+\beta \underline{I}$ is a spherical zero of $f(x)$, then we have $f(w)=w A(w)+$ $B(w)=0$. According to Corollary 2.1, we also have $f(\bar{w})=\bar{w} A(\bar{w})+B(\bar{w})=0$. Noting $A(w)=A(\bar{w}), B(w)=B(\bar{w})$, we have $A(w)=B(w)=0$.

Hence $f^{c}(w)=\overline{A(w)} w+\overline{B(w)}=0$.

b) If $w_{1}=\alpha+\beta \underline{I_{1}}$ ( $\beta$ can be zero $)$ is a zero of $f(x)$, then $f\left(w_{1}\right)=w_{1} A\left(w_{1}\right)+B\left(w_{1}\right)=0$. Hence $f^{c}\left(\overline{w_{1}}\right)=\overline{A\left(w_{1}\right)} \overline{w_{1}}+\overline{B\left(w_{1}\right)}=\overline{w_{1} A\left(w_{1}\right)+B\left(w_{1}\right)}=0$. This completes the proof.

Next, we will discuss the following cases:

Case I. If $F(\alpha)=0, \alpha \in \mathbf{R}$, then

$$
F(\alpha)=f(\alpha) f^{c}(\alpha)=f(\alpha) \overline{f(\bar{\alpha})}=f(\alpha) \overline{f(\alpha)}=|f(\alpha)|^{2}=0 .
$$

Hence, $f(\alpha)=0$.

Case II. If $\alpha \pm \mathbf{i} \beta$ are zeroes of $F(z)$, according to Theorem 2.4, we have $F(\alpha+\beta \underline{I})=0$ for all $\underline{I} \in S^{m-1}$. If $\alpha+\beta \underline{I}$ satisfies (4), that is

$$
(\alpha+\beta \underline{I}) A(\alpha+\beta \underline{I}) \overline{B(\alpha+\beta \underline{I})}=A(\alpha+\beta \underline{I}) \overline{B(\alpha+\beta \underline{I})}(\alpha+\beta \underline{I}),
$$

we have $F(\alpha+\beta \underline{I})=f(\alpha+\beta \underline{I}) f^{c}(\alpha+\beta \underline{I})=0$. Therefore $f(\alpha+\beta \underline{I})=0$ or $f^{c}(\alpha+\beta \underline{I})=0$. Adding to Lemma 3.2 we obtain that $f(\alpha+\beta \underline{I})=0$ for all $\underline{I} \in S^{m-1}$.

On the other hand, if $f(\alpha+\beta \underline{I})=0$ for all $\underline{I} \in S^{m-1}$, then $f(\alpha+\beta \underline{I}) f^{c}(\alpha+\beta \underline{I})=0=$ $F(\alpha+\beta \underline{I})$. Hence $\alpha+\beta \underline{I}$ satisfies (4).

Case III. If $\alpha \pm \mathbf{i} \beta$ are zeroes of $F(z)$, and for $\underline{I}_{1} \in S^{m-1}, \alpha+\beta \underline{I_{1}}$ satisfies (4), then we have $F\left(\alpha+\beta \underline{I_{1}}\right)=f\left(\alpha+\beta \underline{I_{1}}\right) f^{c}\left(\alpha+\beta \underline{I_{1}}\right)=0$. Therefore $f\left(\alpha+\beta \underline{I_{1}}\right)=0$ or $f^{c}\left(\alpha+\beta \underline{I_{1}}\right)=0$. Adding to Lemma 3.2 we obtain that $f\left(\alpha+\beta \underline{I_{1}}\right)=0$ or $f\left(\alpha-\beta \underline{I_{1}}\right)=0$.

On the other hand, if $f\left(\alpha+\beta \underline{I_{1}}\right)=0$ or $f\left(\alpha-\beta \underline{I_{1}}\right)=0$, then $\alpha+\beta \underline{I_{1}}$ satisfies (4).

Case IV. If $\alpha \pm \mathbf{i} \beta$ are zeroes of $F(z)$ and for all $\underline{I} \in S^{m-1}, \alpha+\beta \underline{I}$ does not satisfy $(4)$, then $f(x)$ has no non-real zeroes.

In all, we have

Theorem 3.1 Let $f(x)=\sum_{n=0}^{\infty} x^{n} a_{n}, a_{n} \in \mathbf{R}_{1}^{m}$, be a slice monogenic function in $B(0, R)$. Then a set of sufficient and necessary conditions for $f$ to have a zero $\alpha+\beta \underline{I}$ ( $\beta$ can be zero) is that $\alpha \pm \mathbf{i} \beta$ are zeroes of $F(z)$ and $\alpha+\beta \underline{I}$ satisfies (4).

Note 3.2 If $A(\alpha+\beta \underline{I}) \overline{B(\alpha+\beta \underline{I})} \in \mathbf{R}_{1}^{m}$, then we can choose $\underline{I_{1}}$ parallel to $\operatorname{Vec}[A(\alpha+$ $\beta \underline{I}) \overline{B(\alpha+\beta \underline{I})}]$ ( denoted by $\underline{I_{1}} \| \operatorname{Vec}[A(\alpha+\beta \underline{I}) \overline{B(\alpha+\beta \underline{I})}]$ ) such that $\alpha+\beta \underline{I_{1}}$ satisfies (4). According to Case III, we have $f\left(\alpha+\beta \underline{I_{1}}\right)=0$ or $f\left(\alpha-\beta \underline{I_{1}}\right)=0$.

Theorem 3.2 Assume that $f(x)=\sum_{n=0}^{\infty} x^{n} a_{n}, a_{n} \in \mathbf{R}_{1}^{m}$, is a slice monogenic function in $B(0, R)$ and $\alpha \pm \mathbf{i} \beta$ are two conjugate zeroes of $F(z)$. If

$$
A(\alpha+\beta \underline{I}) \overline{B(\alpha+\beta \underline{I})} \in \mathbf{R}_{1}^{m},
$$


then $\alpha+\beta \underline{I_{1}}$ or $\alpha-\beta \underline{I_{1}}$ is a zero of $f(x)$, where $\underline{I_{1}} \| \operatorname{Vec}[A(\alpha+\beta \underline{I}) \overline{B(\alpha+\beta \underline{I})}]$.

Note 3.3 In the quaternionic space $H$, it is paravector-valued algebra. For any polynomial $P(q)=q^{n} a_{n}+q^{n-1} a_{n-1}+\cdots+q a_{1}+a_{0}, a_{n} \in H, F(z)=P(z) P^{c}(z)$ always has zeroes and $A(q) \overline{B(q)} \in H$. Therefore, any polynomials $P(q)$ always have zeroes. In [7], they use the similar methods to prove the fundamental theorem for quaternions.

Noting that $A(x)=a_{1}+A_{2}(x) a_{2}+\cdots+A_{n}(x) a_{n}+\cdots, B(x)=a_{0}+B_{2}(x) a_{2}+\cdots+$ $B_{n}(x) a_{n}+\cdots$, we have:

Corollary 3.1 Assume that $f(x)=\sum_{n=0}^{\infty} x^{n} a_{n}, a_{n} \in \mathbf{R}_{1}^{m}$, is a slice monogenic function in $B(0, R)$ and $\alpha \pm \mathbf{i} \beta$ are two conjugate zeroes of $F(z)$. If $a_{0} \in \mathbf{R}_{1}^{m}$ and $a_{1}, a_{2}, \cdots \in \mathbf{R}$, then $\alpha+\beta \underline{I_{1}}$ or $\alpha-\beta \underline{I_{1}}$ is a zero of $f(x)$, where $\underline{I_{1}} \| \operatorname{Vec}\left(a_{0}\right)$.

Corollary 3.2 Assume that $f(x)=\sum_{n=0}^{\infty} x^{n} a_{n}, a_{n} \in \mathbf{R}_{1}^{m}$, is a slice monogenic function in $B(0, R)$ and $\alpha \pm \mathbf{i} \beta$ are two conjugate zeroes of $F(z)$. If $a_{1} \in \mathbf{R}_{1}^{m}$ and $a_{0}, a_{2}, a_{3}, \cdots \in \mathbf{R}$, then $\alpha+\beta \underline{I_{1}}$ or $\alpha-\beta \underline{I_{1}}$ is a zero of $f(x)$, where $\underline{I_{1}} \| \operatorname{Vec}\left(a_{1}\right)$.

\section{References}

[1] F. Brackx, R. Delanghe and F. Sommen, Clifford Analysis, (Research Notes in Mathematics, Vol. 76), Boston, London, Melbourne: Pitman Advanced Publishing Company, 1982.

[2] R. Delanghe, F. Somman and Soucek V, Clifford Algebra and Spinor-Valued Functions, Dorderecht, Boston, London: Kluwer Academic Publishers, 1992.

[3] I. Niven, Equations in quaternions, Amer. Math. Monthly, 48 (1941), 654-661.

[4] I. Niven, The roots of quaternions, Amer. Math. Monthly, 49 (1942), 386-388.

[5] S. Eilenberg and I. Niven, The fundamental theorem of algebra for quaternions, Bull. Amer. Math. Soc., 50 (1994), 246-248.

[6] A. Pogorui and M. Shapiro, On the structure of the set of zeroes of quaternionic polynomials, Complex Variables, 49(6) (2004), 379-389.

[7] G. Gentili and C. Stoppato, Zeroes of regular functions and polynomials of a Quaternionic variable, Michigan Math. J., 56(2008), 655-667.

[8] Graziano Gentili, Daniele C. Struppa and Fabio Vlacci, The fundamental theorem of algebra for Hamilton and Cayley numbers, Mathematische Zeitschrift, 259 (4) (2008), 895-902. 
[9] Graziano Gentili and Daniele C. Struppa, On the multiplicity of zeroes of polynomials with quaternionic coefficients, Milan Journal of Mathematics, 76(1) (2008), $15-25$.

[10] B. Datta and S. Nag, Zero-sets of quaternionic and octonionic analytic functions with central coefficients, Bulletin of the London Mathematical Society, 19(4) (1987), 329-336.

[11] F. Colombo, I. Sabadini and D. C. Struppa, Slice monogenic functions, Israel Journal of Mathematics, 171(1) (2009), 385-403.

[12] F. Colombo, I. Sabadini and D. C. Struppa, An extension theorem for slice monogenic functions and some of it consequences, to appear in Israel Journal of Mathematics.

[13] Y. Yang and T. Qian, On sets of zeroes of Clifford algebra-valued polynomials, to appear in Acta Mathematica Scientia.

[14] Y. Yang and T. Qian, On sets of zeroes of Clifford Analytic Functions with Real Coefficients, to appear.

[15] F. Sommen, Some connections between Clifford analysis and complex analysis, Complex Variables Theory Appl, 1(1) (1982), 97-118

[16] A. McIntosh, Clifford algebras, Fourier theory, singular integrals, and harmonic functions on Lipschitz domains, In: Clifford Algebras in Analysis and Related Topics (Fayetteville, Ark, 1993), Boca Raton, Florida: Stud. Adv. Math. CRC press, 1996, 33-87

[17] H. Leutwiler Modified Clifford Analysis, Complex Variables Theory Appl, 17 (1992), 153-171.

[18] C. G. Cullen, An integral theorem for analytic intrinsic functions on quaternions, Duck Math. J., 32 (1965), 139-148.

[19] Gentili G. and Struppa D. C. A new approach to Cullen-regular functions of a Quaternionic variable, C. R. Math. Acad. Sci. Paris, 342 (2006), 741-744

[20] G. Gentili and D. C. Struppa, A new theory of regular functions of a quaternionic variable, Adv. Math., 216 (2007), 279-301. 